EPJ Web of Conferences 41, 02024 (2013)

DOI: $10.1051 /$ epjconf/20134102024

(C) Owned by the authors, published by EDP Sciences, 2013

\title{
Temporal and spectral evolution of an interrupted virtual single-photon transition: creation of optical gain and loss
}

\author{
Jens Herrmann ${ }^{1}$, Matthias Weger ${ }^{1}$, Reto Locher ${ }^{1}$, Mazyar Sabbar ${ }^{1}$, Paula Rivière ${ }^{2,3}$, Ulf Saalmann ${ }^{3}$, \\ Jan-Michael Rost ${ }^{3}$, Lukas Gallmann ${ }^{1}$, and Ursula Keller ${ }^{1}$ \\ ${ }^{1}$ Physics Department, ETH Zurich, CH-8093 Zürich, Switzerland \\ ${ }^{2}$ Departamanto de Química, Universidad Autónoma de Madrid, ES-28049 Madrid, Spain \\ ${ }^{3}$ Max Planck Institute for the Physics of Complex Systems, D-01187 Dresden, Germany
}

\begin{abstract}
We examine the optical response of a virtual dipole transition of a quantum mechanical two-level system (TLS). In the case of off-resonant excitation the timeintegrated dipole response (TIDR) is expected to be zero, which corresponds to transparency of the system with respect to the exciting pulse. Our new time-frequency representation reveals that even for a zero TIDR there are positive and negative contributions included in the response. Furthermore, we present a way to access these contributions by using a second electromagnetic field, which interrupts the temporal evolution of the dipole response. The theoretical results are confirmed by attosecond transient absorption spectroscopy in helium $(\mathrm{He})$.
\end{abstract}

\section{Introduction}

The advent of light pulses with sub-femtosecond duration opened the door to experiments, which are able to follow the electronic dynamics of atoms and molecules [1-5]. In attosecond transient absorption spectroscopy a single attosecond pulse (SAP) or an attosecond pulse train (APT) is overlapped in an interaction target with a second laser pulse, which usually is the fundamental infrared (IR) pulse. The transmitted photon yield in the extreme ultraviolet (XUV) spectral region is detected as a function of photon energy and delay between the two pulses. Most of the experiments performed so far concentrate on the excitation of bound-bound transitions in matter or the direct ionization. The off-resonant excitation of matter was not investigated in previous attosecond transient absorption spectroscopy experiments.

Here, we present a novel time-frequency representation of the temporal evolution of an offresonantly excited virtual dipole transition. The temporal evolution is not accessible in linear optical experiments where only the TIDR can be detected. Our theoretical analysis reveals positive and negative contributions, which are inherently included in the response. In the case of off-resonant excitation these contributions cancel out in the time integration. A second electromagnetic field can interrupt the evolution in time. This results in a nonzero and therefore detectable TIDR.

We verify the theoretical results with an attosecond transient absorption experiment in He, where we observe optical gain and loss of equal magnitude. The optical gain and loss are gated in time by the delay between the pulses.

This is an Open Access article distributed under the terms of the Creative Commons Attribution License 2.0, which permits unrestricted use, distribution, and reproduction in any medium, provided the original work is properly cited. 


\section{EPJ Web of Conferences}

\section{Results}

For our theoretical time-frequency analysis we use a quantum-mechanical TLS, consisting of the ground state $|g\rangle$ and the excited state $|e\rangle$. Both states are separated in energy by the transition energy $\Delta$. The response of the system is characterized by its transition dipole $d(t) \propto a(t)+a^{*}(t)$, where $a(t)$ represents the amplitude of the excited state $|e\rangle$. If we excite the TLS off-resonantly, the transition dipole can be written in rotating-wave approximation as (using atomic units):

$$
d(t)=\frac{1}{\widetilde{\omega}-\Delta} \tilde{V}(t)
$$

Here $\widetilde{V}(t)$ and $\widetilde{\omega}$ are the electric field and the angular frequency of the driving field. Figure 1A shows the temporal evolution of the dipole as a function of time and photon energy. The exciting pulse at $23.37 \mathrm{eV}$ is off-resonant with respect to the excited state at $23.09 \mathrm{eV}$. A second electromagnetic field is now used to modulate the transition energy of the system and interrupt the temporal evolution of the dipole. The interruption results in a nonzero TDIR since positive and negative contribution do not cancel out anymore. A positive response corresponds to absorption, while a negative response represents a net emission of photons, which can be understood as optical gain. Figure 1B displays the TIDR as a function of photon energy and delay.

A

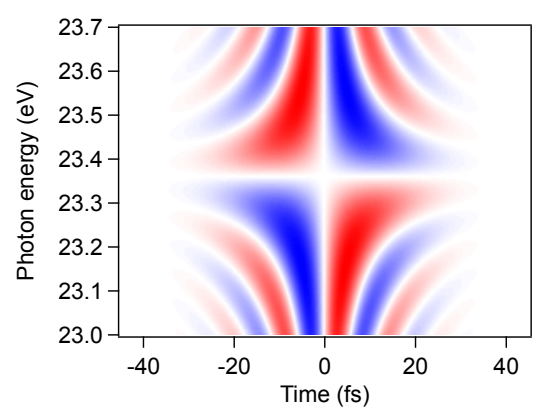

B

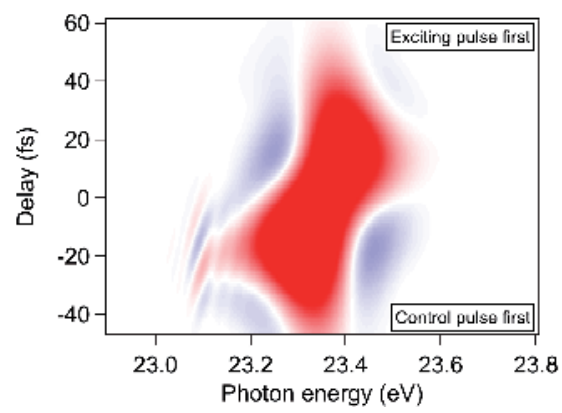

Fig. 1. Figure 1A shows the temporal evolution of the dipole response in time-frequency representation. Light grey (red) corresponds to a positive response and dark grey (blue) to a negative response. In Figure 1B the calculated time-integrated dipole response for different values of the delay is displayed.

We test our model by comparing it with attosecond transient absorption spectroscopy in He. The He $1 \mathrm{~s}^{2}$ ground state represents the lower state of the TLS, while the excited state $|e\rangle$ corresponds to the excited $1 \mathrm{~s} 3 \mathrm{p}$ state. For the off-resonant excitation we use the 15 th harmonic of an APT. It is energetically centered between the $1 \mathrm{~s} 3 \mathrm{p}$ and $1 \mathrm{~s} 4 \mathrm{p}$ excited state. The latter is negligible since its transition strength is significantly weaker compared to the $1 \mathrm{~s} 3 \mathrm{p}$ transition. For the control pulse we use a moderately strong (intensity $<10^{13} \mathrm{~W} / \mathrm{cm}^{2}$ ) IR pulse. This control pulse can be delayed in time by changing the optical path length. 


\section{XVIIIth International Conference on Ultrafast Phenomena}

The transmitted XUV yield shows a strong decrease around $\sim 0$ fs delay. This relates to a twophoton absorption of one XUV and one IR photon (see Figure 2A). In addition the photon energy is sufficient to ionize He. Figure 2B shows the change of absorbance. Despite the strong absorption feature (positive change of absorbance), which was already visible in the XUV yield, we now observe a net emission of XUV photon (negative change) for certain photon energies and delay between the pulses. In excellent qualitative agreement with the theoretical results, both, absorption and optical gain are of the same order of magnitude.

A

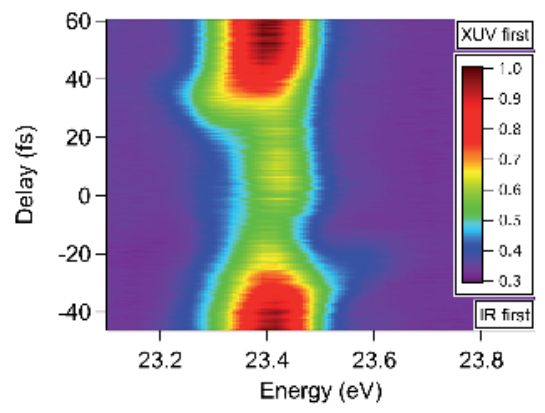

B

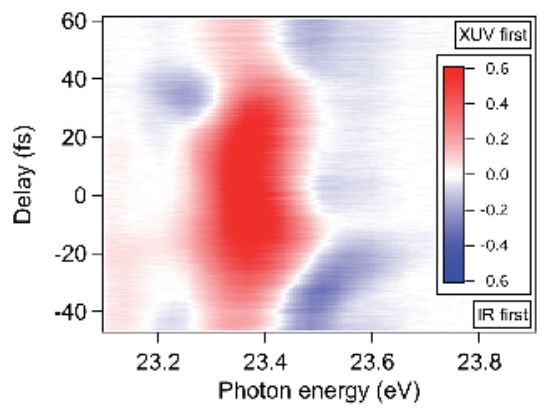

Fig. 2. Figure $2 \mathrm{~A}$ shows the transmitted XUV radiation as a function of delay and photon energy. Around $0 \mathrm{fs}$ delay $50 \%$ of the XUV radiation is absorbed due to two-photon absorption. Figure 2B depicts the change of absorbance as a function of delay and photon energy.

\section{Conclusion}

We introduced a new time-frequency representation for the temporal evolution of a virtual dipole transition. This representation shows that even in the case of a zero TIDR there exist positive and negative contributions to the response. Furthermore, we demonstrated for the first time that a second electromagnetic field can interrupt the dipole response, which leads to optical gain and loss. The delay between the two fields enables us choose between gain and loss at certain XUV photon energies. Finally, we experimentally verify our theoretical results with attosecond transient absorption spectroscopy in He.

\section{References}

1. M. Hentschel, R. Kienberger, C. Spielmann, G. A. Reider, N. Milosevic, T. Brabec, P. Corkum, M. Drescher, F. Krausz, Nature 414, 509 (2001)

2. P. M. Paul, E. S. Toma, P. Breger, G. Mullot, F. Augé, P. Balcou, H. G. Muller, P. Agostini, Science 292, 1689 (2001)

3. E. Goulielmakis, Z.-H. Loh, A. Wirth, R. Santra, N. Rohringer, V. S. Yakovlev, S. Zherebtsov, T. Pfeifer, A. M. Azzeer, M. F. Kling, S. R. Leone, F. Krausz, Nature 466, 739 (2010)

4. H. Wang, M. Chini, S. Chen, C.-H. Zhang, F. He, Y. Cheng, Y. Wu, U. Thumm, Z. Chang, Phys. Rev. Lett. 105, 143002 (2010)

5. M. Holler, F. Schapper, L. Gallmann, U. Keller, Phys. Rev. Lett. 106, 123601 (2011) 